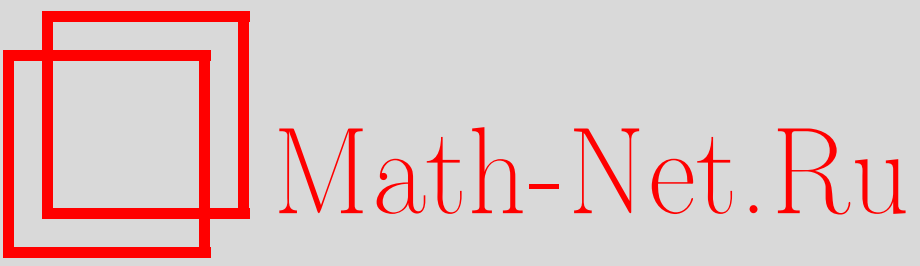

Т. Д. Вентцель, Ю. А. Дубинский, А. М. Ильин, В. А. Кондратьев, В. П. Михайлов, А. А. Мальцев, О. А. Олейник, С. И. Похожаев, Н. Х. Розов, Г. А. Чечкин, T. А. Шапошникова, Анатолий Сергеевич Калашников (некролог), УМH, 2000, том 55, выпуск 5, 161-168

DOI: https://doi.org/10.4213/rm322

Использование Общероссийского математического портала Math-Net.Ru подразумевает, что вы прочитали и согласны с пользовательским соглашением

http://www.mathnet.ru/rus/agreement

Параметры загрузки:

IP : 3.85 .7 .115

26 апреля 2023 г., 02:33:19 


\section{АНАТОЛИЙ СЕРГЕЕВИЧ КАЛАШНИКОВ}

29 февраля 2000 года скончался профессор Московского государственного университета Анатолий Сергеевич Калашников. Мы потеряли замечательного человека, видного ученого, талантливого педагога, человека редкостного трудолюбия и скромности, ответственности за каждое дело, человека, работать с которым было радостью.

Анатолий Сергеевич Калашников родился 30 июня 1934 года в Москве. Окончив механико-математический факултет МГУ (1956), он поступил в аспирантуру Математического института им. В.А. Стеклова АН СССР, где его научным руководителем была Ольга Арсеньевна Олейник. По окончании аспирантуры (1959) Анатолий Сергеевич был приглашен на работу на механико-математический факультет МГУ, сначала на кафедру математического анализа, затем (1964) - на кафедру дифференциальных уравнений, с которой

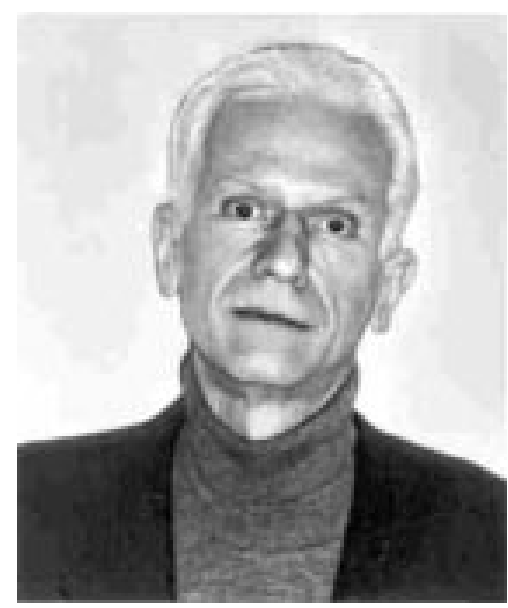
не расставался уже до конца.

В 1960 году Анатолий Сергеевич защитил кандидатскую диссертацию "О разрывных решениях квазилинейных уравнений и систем гиперболического типа". На факультете читал курсы уравнений математической физики, уравнений с частными производными, специальные курсы для студентов. Постоянно руководил спецсеминарами, курсовыми и дипломными работами, аспирантами. Под его руководством защищено 9 кандидатских диссертаций. Его докторская диссертация "Краевые задачи с факторами усиления и ослабления нелинейных эффектов" была отмечена ВАК как одна из самых силњых диссертаций года.

Анатолий Сергеевич не ограничивался только работой в МГУ, он поддерживал тесные связи с другими вузами города Москвы, неоднократно выступал с докладами в Московском энергетическом институте, Московском государственном техническом университете им. Н.Э. Баумана и других.

Научные исследования Анатолий Сергеевич вел по многим направлениям. Им найдены точные классы однозначной разрешимости задачи без начальных данных для линейных вырождающихся гиперболических уравнений с неограниченной областью зависимости, для линеаризованной системы уравнений вращающейся жидкости, для линейных интегро-дифференциалњнтх уравнений с операторами Вольтерра типа свертки им указаны новые виды условий на бесконечности, выделяюших единственное решение линейного уравнения произвольного порядка и типа с постоянными коэффициентами во всем пространстве; в задаче Коши для нелинейных вырождающихся параболических уравнений получены необходимые и достаточные условия наличия бесконечной, конечной и нулевой скорости распространения возмущений, их пространственной 
и временной локализации, мгновенной компактификации носителя решения; в краевых задачах с растущими данными для нескольких классов нелинейных эволюционных уравнений и систем установлены критерии внутренней ограниченности решений; для ряда возникающих в прикладных задачах эволюционных и стационарных уравнений, которые содержат искомые решения под знаками степенных функций с малым параметром в показателях, выведены точные условия, гарантирующие сохранение нелинейных эффектов при стремлении малого параметра к нулю.

Большое количество статей Анатолия Сергеевича посвящено нелинейным эффектам, характерным для параболических уравнений. Со времен классической статьи Я.Б. Зельдовича и А. С. Компанейца (1950) известно, что свойства решений нелинейных параболических уравнений сильно отличаются от свойств решений обычного линейного уравнения теплопроводности. Например, нелинейное уравнение вида

$$
D_{t} u-a D_{x}^{2}\left(u^{m} \operatorname{sgn} u\right)+b|u|^{p} \operatorname{sgn} u=0, \quad m>0, \quad p>0, \quad a>0,
$$

вырождающееся при $u=0$, описывает разные физические процессы: фильтрацию жидкости через пористую среду, обтекание твердого тела вязкой жидкостью, процессы теплопроводности или диффузии в среде, параметры которой зависят от температуры; оно же описывает динамику биологических популяций.

Для случаев, когда это уравнение описывает модель диффузии, при стремлении решения к нулю случай $0<m<1$ соответствует быстрой диффузии, случай $m=1$ - нормальной диффузии, а случай $m>1$ - медленной диффузии.

Уравнение, вообще говоря, не имеет гладких решений и его решения понимаются в том или ином обобщенном смысле.

Можно перечислить ряд специфических нелинейных эффектов для решений указанного уравнения при $m \neq 1$ (для простоты ниже рассматриваются неотрицательные решения уравнения).

1. При медленной диффузии $(m>1)$ скорость распространения возмущений конечна. Это значит, что если начальное значение решения $u(x, 0)=f(x)$ таково, что $f(x)=0$ при $x>0$ и $f(x)>0$ при $x<0$, то решение при всех положительных значениях времени финитно справа по $x$.

2. Задержка фронта. Если начальная функция $f(x)$ при стремлении $x$ к нулю слева стремится к нулю достаточно быстро, то для достаточно малых положителњњых $t$ решение $u(x, t)=0$ для $x>0$, т.е. фронт возмущения какое-то время не движется.

3. Локализация по времени. Рассматриваются неотрицательные решения уравнения при $b=$ 0 в случае быстрой диффузии $(0<m<1)$ в полуполосе $\{|x|<l, t \geqslant 0\}$ при нулевых граничных условиях. Тогда существует $T>0$ такое, что $u(x, t)=0$ для всех $t>T$.

4. Локализация по глубине. Рассматривается случай $m>1, b>0,0<p<m$. Тогда если начальная функция финитна справа, то решение при всех значениях времени также финитно справа.

5. Мгновенное сжатие носителя. Пусть $m \geqslant 1, b>0,0<p<1$ и начальная функция стремится к нулю на положительной бесконечности. Тогда при любом положительном значении времени решение финитно справа, и имеет место также локализация по времени.

6. Отсутствие глобальной разрешимости. В случае "суперлинейных источников", т.е. при $b<0, p>1$, в некоторый конечный момент времени решение стремится к бесконечности на некотором множестве значений пространственной переменной.

7. Внутренняя ограниченность. Рассматривается решение уравнения с $m>1$ в положительной четверти плоскости с начальными и граничными условиями, причем граничное значение стремится к бесконечности при $t \rightarrow T$. Если это стремление к бесконечности не слишком быстро, то решение остается ограниченным при $t \rightarrow T$ для всех $x \geqslant \rho, \rho>0$.

Изменение параметров задачи в ту или иную сторону способствует либо усилению, либо ослаблению указанных нелинейных эффектов. Анатолий Сергеевич рассматривает в серии своих работ и в докторской диссертации различные более тонкие факторы усиления и ослабления нелинейных эффектов. В ряде работ рассматриваются уравнения с коэффициентами и даже показателями степени, зависящими от $\boldsymbol{x}$ и $\boldsymbol{t}$. Это значит, что рассматриваются процессы, протекающие в 
неоднородной среде. Коэффициенты могут стремиться к нулю или бесконечности при некоторых значениях времени или пространственной переменной. В задачах обычно присутствуют факторы, усиливающие нелинейный эффект, и одновременно факторы, его ослабляющие. Находятся соотношения меж ду параметрами задачи, гарантирующие наличие или отсутствие исследуемого эффекта. В большинстве случаев получены точные границы для данных задачи, будь то коэффициенты или характер нелинейностей, входящих в уравнение. При построении примеров, доказывающих точность полученных условий, Калашников мастерски строит явные решения уравнений и применяет самые тонкие теоремы сравнения. В ряде других работ рассматриваются нелинейные системы уравнений специального вида и для отдельных компонент их решений исследуются нелинейные эффекты, аналогичные $1-7$.

Значительное число работ посвящено уравнениям, в которых нелинейности содержат параметр $\varepsilon \geqslant 0$. При положительных значениях параметра изучаемые нелинейные эффекты имеют место, а при $\varepsilon \rightarrow 0$ значения параметров стремятся к критическим, для которых эффект пропадает. Также исследуются условия, при которых нелинейный эффект имеет "равномерный по $\varepsilon$ " характер. Анатолий Сергеевич впервые рассмотрел задачу Коши для уравнения

$$
u_{t}+(\varphi(u))_{x}=\varepsilon u_{x x}
$$

с невыпуклой функцией $\varphi(u)$ и обосновал предельный переход при $\varepsilon \rightarrow 0$.

Анатолий Сергеевич является одним из авторов шшроко известной работы “Линейные уравнения второго порядка параболического типа" (УМН. 1962. Т. 17. Вып. 3. С. 3-146), в которой были систематически изложены разнообразные результаты по линейному параболическому уравнению 2-го порядка. Ссылки на эту статью содержатся в большом числе работ по этой тематике.

Хочется отметить колоссальный вклад А. С. в организацию ежегодных совместных заседаний семинара им. И. Г. Петровского и Московского математического общества (конференций Петровского). Его участие существенно выходило за рамки просто формального, он был ответственным за приглашение ученых, составление программы, за сбор и подготовку публикации тезисов, а в последние годы еще и курировал работу оргкомтета.

А. С. много лет вел спецсеминар для студентов и аспирантов. Он был прекрасным учителем и необыкновенно щедро отдавал свои идеи ученикам. Но он учил их не только математике. Общаясь с ним, люди учились простым, но чрезвычайно важным нравственным правилам: нельзя обманывать, нельзя халтурить, нельзя, чтобы твои поступки вредно отражались на других людях. Когда он выходил к доске и начинал писать своим красивым крупным почерком, становилось ясно, что все будет понятно, основательно и надежно.

А. С. был вообще удивительно надежным человеком. Если им было что-то обещано или просто взято под контроль, можно было больше не возвращаться к этому вопросу. Была полная уверенность, что все будет выполнено в срок и с отменным качеством. Он умудрялся при этом тактично, ненавязчиво и незаметно напоминать и другим о деле, если выполнение зависело не только от него. При этом казалось, что часть работы, которую делал кто-то из коллег, делается по инициативе самого исполняюшего, у которого, впоследствии, оставалось приятное чувство сопричастности к прекрасно выполненному делу.

Общаться с Анатолием Сергеевичем всегда было очень приятно. Он был очень хорошим собеседником с тонким чувством юмора. Играл хорошо в шахматы, любил обсуждать решения трудных задач. Старался все время сеять добро вокруг.

Мы лишились замечательного друга, талантливого преподавателя и большого ученого.

Т. Д. Вентцель, Ю. А. Дубинский, А. М. Ильин, В. А. Кондратьев, А.А. Мальиев, В. П. Михайлов, О. А. Олейник, С. И. Похохаев, Н. Х. Розов, Г. А. Чечкин, Т. А. Шапошникова 


\section{СПИСОК НАУЧНЫХ ТРУДОВ А. С. КАЛАШНИКОВА}

[1] О растущих решениях линейных уравнений второго порядка с неотрицательной характеристической формой // Матем. заметки. 1968. Т. 3. № 2. С. 171-178

[2] О линейных вырождающихся параболических уравнениях произвольного порядка с конечной областью зависимости // Матем. заметки. 1969. Т. 6. № 3. С. 289-294

[3] О неограниченных решениях некоторых линейных вырождающихся параболических систем второго порядка // УМН. 1970. Т. 25. № 2. С. 270-272

[4] Задача без начальных условий в классах растуших функций для некоторых линейных вырождающихся параболических систем второго порядка. I // Вестник МГУ. Сер. 1. Матем. Mex. 1971. № 2. C. 42-48

[5] Задача без начальных условий в классах растуших функций для некоторых линейных вырождающихся параболических систем второго порядка. II // Вестник МГУ. Сер. 1. Матем. Mex. 1971. № 3. C. 3-9

[6] О линейных вырождающихся гиперболических уравнениях второго порядка с бесконечной областью зависимости // УМН. 1971. Т. 26. № 4. С. 231-232

[7] О некоторых задачах для линейных уравнений в частных производных с постоянными коэффициентами во всем пространстве и для одного класса в полупространстве // Матем. сб. 1971. T. 85. № 2. C. 189-200

[8] Задача без начальных условий для линейных вырождающихся гиперболических уравнений второго порядка с бесконечной областью зависимости // Матем. сб. 1972. Т. 88. № 4 . C. $609-622$

[9] Об уравнениях типа нестационарной фильтрации с бесконечной скоростью распространения возмущений // Вестник МГУ. Сер. 1. Матем. Мех. 1972. №6. С. 45-49

[10] О задаче Коши в классах растущих функций для некоторых квазилинейных вырождающихся параболических уравнений второго порядка // Дифференц. уравнения. 1973. Т. 9. № 4. C. $682-691$

[11] О семинаре им. И. Г. Петровского // УМН. 1973. Т. 28. №5. С. 257-266 (совм. с О. А. Олейник)

[12] Об условиях единственности обобщенного решения задачи Коши для одного класса квазилинейных вырожддающихся параболических уравнений // Дифференц. уравнения. 1973. Т. 9. № 12 . C. $2207-2212$

[13] О дифференциальных свойствах обобщенных решений уравнений типа нестационарной фильтрации // Вестник МГУ. Сер. 1. Матем. Мех. 1974. № 1. С. 62-68

[14] О характере распространения возмущений в задачах нелинейной теплопроводности с поглощением // ЖВМ и МФ. 1974. Т. 14. № 4. С. 891-905

[15] О характере распространения возмущений в процессах, описываемых квазилинейными вырождающимися параболическими уравнениями // Труды семинара им. И.Г. Петровского. 1975. T. 1. C. $135-144$

[16] О влиянии поглощения на распространение тепла в среде с теплопроводностью, зависящей от температуры // ЖВМ и МФ. 1976. Т. 16. № 3. С. 689-696

[17] Классы единственности решений задачи Коши для линеаризованной системы уравнений вращающейся жидкости // УМН. 1976. Т. 31. № 4. С. 263-264

[18] Всесоюзная конференция по уравнениям с частными производными, посвященная 75-летию со дня рождения И. Г. Петровского // УМН. 1976. Т. 31. № 5. С. 257-263

[19] О непрерывной зависимости обобщенных решений уравнения нестационарной филтрации от функции, задающей режим потока // Прикл. матем. мех. 1978. Т. 42. № 1. С. 183-185

[20] О характере распространения возмущений в нелинейных нестационарных задачах теории теплопроводности и теории фильтрации // Труды Всесоюзной конференции по уравнениям с частными производными, 1978. С. 326-327

Начало списка см. "Математика в СССР (в двух томах) 1958-1967." М.: Наука, Главная редакция физико-математической литературы, 1969. 
[21] О единственности решений интегро-дифференциальных уравнений типа Вольтерра / / УМН. 1978. T. 33. № 3. C. 150-151

[22] Об одном нелинейном уравнении, возникающем в теории нестационарной фильтрации // Труды семинара им. И. Г. Петровского. 1978. Т. 4. С. 137-146

[23] О понятии конечной скорости распространения возмущений // УМН. 1979. Т. 34. № 2 . C. $199-200$

[24] Классы единственности для интегро-дифференциальных уравнений с операторами Вольтерра типа свертки // Функц. анализ и его прилож. 1979. Т. 13. № 2. С. 83-84

[25] Об уравнении теплопроводности с дальнодействием // Дифференц. уравнения. 1979. Т. 15. № 9. С. $1653-1660$

[26] О некоторых свойствах решений второй краевой задачи для параболических уравнений // УМH. 1980. Т. 35. № 4. С. 166

[27] О квазилинейных вырождающихся параболических уравнениях с конечной скоростью распространения возмущений // Дифференциальные уравнения с частными производными. Новосибирск: Наука, 1980. С. 80-83

[28] О задаче Коши для вырождающихся параболических уравнений второго порядка с нестепенными нелинейностями // Труды семинара им. И. Г. Петровского. 1981. Т. 6. С. 83-96

[29] Об эволюции профилей обобщенных решений квазилинейных вырождающихся параболических уравнений // УМН. 1981. Т. 36. № 4. С. 212

[30] О задаче Коши для одного класса квазилинейных вырождающихся параболических уравнений второго порядка // Граничные задачи математической физики. Киев: Наукова думка, 1981. C. $47-48$

[31] Об условиях положительности и ограниченности решений второй краевой задачи для параболических уравнений // Сиб. матем. журн. 1981. Т. 22. № 6. С. 72-80

[32] О распространении возмущений в первой краевой задаче для вырождающегося параболического уравнения двойной нелинейности // Труды семинара им. И. Г. Петровского. 1982. T. 8. C. $128-134$

[33] О характере движения фронтов при нестационарной фильтрации жидкостей, не подчиняющихся закону Дарси // УМН. 1982. Т. 37. № 4. С. 118-119

[34] Об уравнениях нелинейной теории теплопроводности // УМН. 1982. Т. 37. № 5. С. 221-222

[35] Об уравнении теплопроводности в среде с неравномерно распределенными нелинейными источниками или поглотителями тепла // Вестник МГУ. Сер. 1. Матем. Мех. 1983. №3. C. $20-24$

[36] О распространении тепла в неоднородных нелинейных средах // УМН. 1983. Т. 38. № 4. C. 164

[37] О распространении тепла при сильно нестационарных режимах // УМН. 1984. Т. 39. № 4. C. 117

[38] О распространении возмущений в процессах, описываемых вырождающимися параболическими уравнениями с нестепенными нелинейностями // Труды семинара им. И.Г. Петровского. 1984. Т. 10. С. $118-134$

[39] О зависимости свойств решений параболических уравнений в неограниченных областях от поведения коэффициентов на бесконечности // Матем. сб. 1984. Т. 125 (167). № 3 (11). C. $398-409$

[40] О характере распространения тепла в нелинейных средах с существенно нестационарными свойствами // Вестник МГУ. Сер. 1. Матем. Мех. 1985. № 4. С. 34-38

[41] О совместном влиянии нелинейности и нестационарности среды на процесс теплопередачи // УМH. 1985. T. 40. № 5. С. 201-202

[42] О влиянии роста граничных данных на поведение температуры нелинейной нестационарной среды при больших значениях времени // Вестник МГУ. Сер. 1. Матем. Мех. 1986. № 2. C. $40-45$

[43] О поведении решений некоторых неавтономных квазилинейных параболических систем при неограниченном возрастании времени // УМН. 1986. Т. 41. № 4. С. 173 
[44] О некоторых моделях динамики конкурирующих биологических видов // УМН. 1986. Т. 41. № 5. C. $215-216$

[45] О некоторых вопросах качественной теории нелинейных вырождающихся параболических уравнений // Тезисы докладов Всесоюзной конференции “Дифференциальнте уравнения и их приложения". Ашхабад, 1986. С. 87-88

[46] Некоторые вопросы качественной теории нелинейных вырождающихся параболических уравнений второго порядка // УМН. 1987. Т. 42. №2. С. 135-176

[47] Об уравнениях нестационарной фильтрации // Проблемы теории фильтрации и механика процессов повышения неффтеотдачи. М.: Наука, 1987. С. 116-123 (совм. с О.А. Олейник)

[48] О некоторых нелинейных системах, описывающих динамику конкурирующих биологических видов // Матем. сб. 1987. Т. 133 (175). № 1 (5). С. 11-24

[49] Об условиях глобальной ограниченности обобщенных решений задачи Коши для некоторых квазилинейных вырождающихся параболических систем // УМН. 1987. Т. 42. № 4. С. 155

[50] О некоторых системах типа "реакция-диффузия" с нестепенными нелинейностями // Тезисы докладов VI Республиканской конференции "Нелинейные задачи математической физики". Донецк, 1987. С. 55

[51] О распространении тепла в линейных и нелинейных средах с сильно нестационарными свойствами // Труды семинара им. И.Г. Петровского. 1987. Т. 12. С. 137-148

[52] Об одном классе квазилинейных вырождающихся параболических систем // Современные проблемы математической физики. Т. 1. Тбилиси: Изд-во Тб. ун-та, 1987. С. 254-261

[53] Об одном классе систем типа "реакция-диффузия" // Труды семинара им. И. Г. Петровского. 1989. Т. 14. С. $78-88$

[54] О мгновенной компактификации носителей компонент решений некоторых систем типа "реакция-диффузия" // УМН. 1989. Т. 44. № 4. С. 210-211

[55] О свойствах решений некоторых систем класса "реакция-диффузия", содержащих нестепенные нелинейные функции // Тезисы докладов Всесоюзной конференции "Нелинейные проблемы дифференциальных уравнений и математической физики”. Часть 1. Тернополь, 1989. C. $177-178$

[56] О задаче Коши для полулинейных параболических уравнений в классах функций со скачками // Доклады расширенных заседаний семинара ИПМ им. И.Н. Векуа и Тбилисского университета. 1989. Т. 4. № 1. С. 56-59

[57] Об условиях мгновенной компактификации носителей решений полулинейных параболических уравнений и систем // Матем. заметки. 1990. Т. 47. № 1. С. 74-80

[58] О поведении при больших значениях времени некоторых нелинейных систем дифференциально-функциональных уравнений // УМН. 1990. Т. 45. № 4. С. 120

[59] О диффузии смесей при наличии дальнодействия // ЖВМ и МФ. 1991. Т. 31. № 3. С. 424-435

[60] О некоторых нелинейных системах, описьвающих взаимодействие подвижного ресурса с неподвихным потребителем // Тезисы докладов VIII Республиканской конференции "Нелинейные задачи математической физики и задачи со свободной границей”. Донецк: ИПММ AH CCCP, 1991. C. 53

[61] О системах класса "реакция-диффузия" с неограниченными функциями в краевых условиях // Вестник МГУ. Сер. 1. Матем. Мех. 1991. №6. С. 17-22

[62] О взаимодействии факторов роста и диссипации в нестационарных краевых задачах // УМН. 1991. Т. 46. №6. С. 180-181

[63] О поведении вблизи начальной гиперплоскости решений задачи Коши для параболических систем с нелинейной диссипацией // Труды семинара им. И. Г. Петровского. 1992. Т. 16. C. $106-113$

[64] О нелинейных явлениях нестационарных процессов, описываемых асимптотически линейными уравнениями // Дифференц. уравнения. 1993. Т. 29. № 3. С. 381-391

[65] On the interaction between dissipation and supply agents in some nonlinear evolution problems // J. Partial Differential Equations. 1993. V. 6. № 2. P. 108-116 
[66] О квазилинейных вырождающихся параболических уравнениях с сингулярными младшими членами и растущими начальными данными // Дифференц. уравнения. 1993. Т. 29. №6. C. 999-1009

[67] О параболических уравнениях с нелинейностями, исчезающими на бесконечности // УМН. 1993. Т. 48. № 4. C. 182

[68] О некоторых нелинейных математических моделях теории теплопроводности, содержащих малый параметр в показателях // Дифференц. уравнения. 1994. Т. 30. №6. С. 1039-1049

[69] О возмущении критических показателей в некоторых нелинейных задачах математической физики // Докл. РАН. 1994. Т. 337. № 3. С. 320-322

[70] О критических показателях в нелинейных задачах математической физики // УМН. 1994. T. 49. № 4. С. 98

[71] О нелинейных эффектах при распространении тепла в средах с источниками или стоками, близкими к линейным // Дифференц. уравнения. 1995. Т. 31. № 2. С. 277-288

[72] О нелинейных вырождающихся параболических уравнениях с показателями, близкими к критическим / / Тезисы докладов международной конференции "Функциональные пространства, теория приближений, нелинейный анализ", посвященной 90-летию С. М. Никольского, Москва, 27 апреля - 3 мая 1995 г. М.: Изд-во ПАИМС, 1995. С. 143-144

[73] О некоторых задачах нелинейной теории теплопроводности с данными, содержащими мальй параметр в показателях // ЖВМ и МФ. 1995. Т. 35. № 7. С. 1077-1094

[74] On diffusion processes in nonlinear media with asymptotically linear sources or sinks // Abstracts of the International Conference "Nonlinear Differential Equations". Kiev, 1995. P. 66

[75] О поведении неограниченных решений квазилинейных вырождающихся параболических уравнений при стремлении показателя роста к критическому значению // УМН. 1995. Т. 50. № 4. C. 91

[76] О задачах математической физики с факторами усиления и ослабления нелинейных эффектов // Материалы международной конференции "Чебышевские чтения", посвященной 175-летию со дня рождения П. Л. Чебышева. Т. 2. М.: Изд-во МГУ, 1996. С. 388

[77] О влиянии дискретизации на нелинейные явления в моделях математической физики // УМН. 1996. Т. 51. № 5. С. 184-185

[78] Ольга Арсеньевна Олейник (к семидесятилетию со дня рождения) // Труды семинара им. И. Г. Петровского. 1996. Т. 19. С. 5-20 (совм. с Арнольдом В.И., Вишиком М.И., Масловым В. П., Никольским С. М., Новиковым С. П.)

[79] О некоторых нелинейных задачах математической физики с показателями, близкими к критическим // Труды семинара им. И.Г. Петровского. 1996. Т. 19. С. 73-98

[80] Nonlinear phenomena in evolution problems with antagonistic agents // Abstracts of the International Conference "Nonlinear Partial Differential Equations". Kiev, August 26-30, 1997. Donetsk, 1997. P. 86-87

[81] О началном скачке свободной границы в краевой задаче для полулинейного уравнения теплопроводности с поглошением // УМН. 1997. Т. 52. № 6. С. 163-164

[82] Instantaneous shrinking of the support for solutions to certain parabolic equations and systems // Rendiconti Lincei: Matematica e Applicazioni. Serie IX. 1997. V. 8. № 4. P. 263-272

[83] О прекращении решений сингулярных нелинейных дифференциальных уравнений и систем // Тезисы докладов Международной конференции "Функциональные пространства. Дифференциальные операторы. Проблемы математического образования", посвященной 75 -летию члена-корреспондента РАН профессора Л. Д. Кудрявцева, Москва, 23-27 марта 1998 г. М.: Изд-во Российского университета дружбы народов. С. 127

[84] О прекращении решений сингулярных нелинейных дифференциальных уравнений и систем // Труды Международной конференции “Функциональные пространства. Дифференциальные операторы. Проблемы математического образования", посвященной 75 -летию члена-корреспондента РАН профессора Л. Д. Кудрявцева, Москва, 23-27 марта 1998 г. Т. 2. М.: Изд-во Российского университета дружбы народов, 1998

[85] Дискретизация и нелинейные эффекты в математических моделях теории теплопроводности // ЖКВМ и МФ. 1998. Т. 38. № 4. С. 600-606 
[86] On quenching of solutions to nonautonomous nonlinear evolution equations and the diffusion quenching phenomenon // Abstracts of the International Conference dedicated to the 90-th Anniversary of L.S. Pontryagin. Volume "Differential Equations". Moscow: Moscow State Univ. Press, 1998. P. 54-55

[87] О наличии начального скачка у температурного фронта при распространении тепла в среде с нелинейными стоками // УМН. 1998. Т. 53. № 4. С. 144

[88] О задаче Коши в классах растущих функций для вырождающихся параболических уравнений с двойными нелинейностями // Фундамент. и прикл. матем. 1998. Т. 4. № 2. С. 543-557

[89] Алексей Федорович Филиппов (в соавторстве) // Дифференц. уравнения. 1998. Т. 34. № 10. C. $1299-1303$

[90] On quenching of solutions to nonautonomous semilinear parabolic equations and reactiondiffusion systems // Appl. Anal. 1999. V. 71. № 1-4. P. 127-138 